\title{
ON A GAP IN THE ORDINARY PRESENTATION OF WEIERSTRASS'S THEORY OF FUNCTIONS.
}

BY PROFESSOR WILLIAM F. OSGOOD.

(Read before the American Mathematical Society, December 28, 1903.)

IN the early days of the development of the calculus, intuition and formalism played an important rôle. The attempt of Lagrange to base the calculus on well defined infinite processes was taken up by Cauchy and Abel and carried through by these men and their successors. Among the latter the name of Weierstrass stands out most prominently. We are in the habit of thinking of this work as completed in all its details, at least so far as fundamental theorems and processes are concerned. In the further development of analysis the theory of functions of a complex variable is believed to be completely arithmetized, and the followers of Weierstrass and Méray point with pride to the fact that, when the theory is based on a single infinite process, namely that of power series, - the process of integration would have served the purpose better, if only one infinite process is to be admitted, - that then intuition has been completely eliminated. But is this really the case? Consider the following theorem.

Theorem (A). Let the power series

$$
\mathfrak{P}(z \mid a)=c_{0}+c_{1}(z-a)+c_{2}(z-a)^{2}+\cdots
$$

define an element of an analytic function and let it be possible to continue this element analytically along any path lying in a certain finite region $S$, inclusive of the boundary; let $S$ be bounded by a single closed curve without multiple points. Then there exists a function $f(z)$ which is single-valued in $S$ and has throughout $S$ the character of an integral function, $i$. e., it can be developed by Taylor's theorem at each point of $S$; and which furthermore coincides with the given element $\mathfrak{P}(z \mid a)$ in the neighborhood of the point $z=a$.

Proofs of this theorem, or of one substantially equivalent to it, are to be found in the treatises that deal with Weierstrass's 
theory of functions.* They all depend essentially on geometric theorems, frequently not even formulated, the truth of which in every case rests on intuition, or rather on analogy, for it is impossible to see what happens in a complicated region bounded even by broken lines; and what is really done is to reason by analogy from the case of squares and circles and such simple figures as these. Now it is a perfectly reasonable point of view to take that, because arithmetization has in the past served a useful end in analysis, it by no means follows that it is desirable to carry the method through consistently to the last detail. Thus the followers of Cauchy and Riemann, who have based the theory of functions on the calculus and thus followed the lines of least resistance, may well take the ground that there are more important things to do in analysis than to arithmetize the geometry of the domain of the independent variables. But for a school to take this stand who for puristic reasons are not willing to admit the process of integration into the theory of functions of a complex variable, appears to be straining at a gnat and swallowing a camel.

We proceed now to a proof of the foregoing theorem in which the geometric theorems employed are proved arithmetically. $\dagger$

$A$ Lemma.-We define a region $\sigma$ by means of the relations $\$$

$$
o<x<a, \quad \phi(x)<y<f(x),
$$

where $f(x), \phi(x)$ are single-valued continuous functions in the complete $\S$ interval $o \leqq x \leqq a$. The regions $\sigma$ that concern us are of three types, indicated by the accompanying figures.

In Type $\mathrm{I}, \phi(x)=0$; in particular, $f(x)$ may be constant, thus giving a rectangle or square. In Type II, $\phi(x)=\lambda x$,

* Cf. Biermann, Analytische Funktionen, pp. 187-188. Jordan, Cours d'analyse, vol. I, 2d ed., 1893, \& 346. Harkness and Morley, Treatise on the Theory of Functions, 1893, \& 127.

$\dagger$ Lest the reader may be on the alert for difficulties which we have avoided from the start through a proper choice of our hypotheses, we will say at this point that our curves and functions have all the continuity requisite for intuitional purposes, and that that which is central in our investigation would remain unaltered if we were to restrict ourselves to broken lines instead of regular curves. But it is just as easy to deal with the latter as with the former. The general Jordan curve nowhere enters into consideration, though many of our results may be extended to such curves without difficulty.

$\ddagger$ The real " $\alpha$ " here used must not be confused with the complex " $a$ " of Theorem (A).

\& Professor Pierpont proposes the term complete as the English equivalent for abgeschlossen. 
where $\lambda$ is a positive constant. Type III is characterized by the relations

$$
f(o)=\phi(o)=0, \quad f^{\prime}(o)=\phi^{\prime}(o) \geqq 0,
$$

where furthermore the points of the line $y=f^{\prime}(a) x$ for which $0<x \leqq a^{\prime}<a$ do not all lie inside of $\sigma$. More generally we admit as regions $\sigma$ all regions obtainable from these three types by rotation through $\pm \pi / 2$, reflection, and translation.

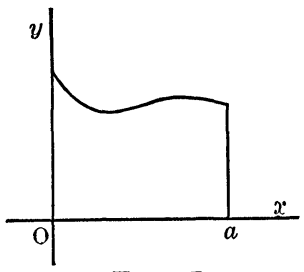

TYPe I.

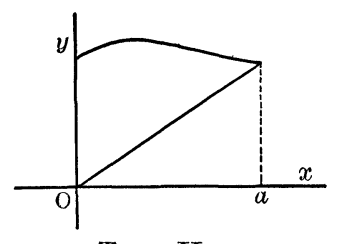

TyPe II.

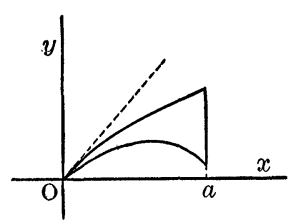

TYPe III.

Arithmetically this means regions whose points $(x, y)$ are obtainable from the points $\left(x^{\prime}, y^{\prime}\right)$ of a region of one of the above types by one of the following transformations:

$$
x-x_{0}=\epsilon x^{\prime}, \quad y-y_{0}=\eta y^{\prime} ; \quad x-x_{0}=\epsilon y^{\prime}, \quad y-y_{0}=\eta x^{\prime},
$$

where $\epsilon$ and $\eta$, independently of each other, take on the values +1 and -1 . The lemma may now be stated as follows.

Let $S$ be a finite region of the plane, whose boundary consists of a finite number of regular closed curves* without multiple points. Then it is possible to divide the region $S$ into a finite number of regions $\sigma$.

The division is effected by laying down a net-work of squares bounded by the lines

$$
x=m / 2^{\mu}, \quad y=n / 2^{\mu}, \quad(m, n=0, \pm 1, \pm 2, \cdots),
$$

* By a regular curve is meant a curve that has a continuously turning tangent, except possibly at a finite number of vertices, and at these points it shall have both a forward and a backward tangent. Such a curve is made up of a finite number of pieces, the points of each of which may be represented arithmetically by the formulas $x=f(t), y=\phi(t)$, where $f(t), \phi(t)$ are, together with their first derivatives, single valued and continuous in the complete interval $t_{0} \leqq t \leqq t_{1}$, and where furthermore $f^{\prime}(t)^{2}+\phi^{\prime}(t)^{2}>0$ in that interval.

The lemma may be stated more generally so as to include the case of boundaries which are made up merely of a finite number of (open or closed) regular curves and which have but a tinite number of multiple points and points of intersection. 
where $\mu$ denotes a positive integer. If the bounding curves have no vertices, only regions $\sigma$ of Type I are needed. An arithmetic proof of this lemma will be found in the writer's forthcoming wrork on the Theory of Functions. *

Proof of Theorem (A) for a Region $\sigma$. Consider the region

$$
\sigma: \quad 0<x<a, \quad 0<y<f(x)
$$

and the path $\Gamma$ consisting of a broken line joining the points $(0,0)$ and $(\xi, \eta)$ of $\sigma$,

$$
\left.\left.\Gamma: \quad \begin{array}{c}
0 \leqq \dot{x} \leqq \xi \\
y=0
\end{array}\right\} \quad \begin{array}{c}
x=\xi \\
0 \leqq y \leqq \eta .
\end{array}\right\}
$$

Let the hypotheses of Theorem (A) be fulfilled for the region $\sigma$ and let $a$ be the point $z=x+y i=0$. Then the element $\mathfrak{P}(z \mid a)$ can be continued analytically along $\Gamma$ to the point $\zeta=\xi+\eta i$ and thus a single-valued function $f(\zeta)$ is defined throughout $\sigma$. That this function is developable by Taylor's theorem at every point of $\sigma$ can be shown at once by the ordinary methods of the theory of functions. In particular it follows from this result that if $\mathfrak{P}(z \mid a)$ be continued analytically round the complete boundary of $\sigma$, this element will come back to its initial value. We note that if $a$ had been any other point of the boundary of $\sigma$, a similar determination of the function $f(z)$ would have been possible. For a region $\sigma$ of Type II or III the process is precisely similar.

An Intermediate Theorem. - We will next show that, under the conditions of Theorem (A), the element $\mathfrak{B}(z \mid a)$, when continued analytically round the boundary of $S$, comes back to its initial value. Let $a$ be a point of the boundary and divide $S$ according to the lemma into a finite number of regions $\sigma$. Let $\sigma_{1}$ be one of these regions, on the boundary of which $a$ lies; suppose $a$ is at a corner of $\sigma_{1}$. We will first sketch the proof and then fill in the details.

Let $S_{1}$ be the part of $S$ that remains when $\sigma_{1}$ is taken away. Since $\mathfrak{P}(z \mid a)$ comes back to its initial value when continued analytically round the boundary of $\sigma_{1}$, it follows that it comes back to the same value when continued round the boundary of $S_{1}$ as when continued round the boundary of $S$. Now repeat the step, proceeding with $S_{1}$ just as a moment ago with $S$. The

* Allgemeine Funktionentheorie, B. G. Teubner's Sammlung von Lehrbüchern auf dem Gebiete der mathematischen Wissenschaften u.s.w. 
new region $S_{2}$ will consist of two regions $\sigma$ fewer than $S$. And so on. Finally only a single region $\sigma$ will remain. But for this region $\mathfrak{P}(z \mid a)$ will come back to its initial value, when continued analytically around the boundary, and thus the theorem is proved.

'Turning now to the development of the details of the proof just sketched, we will first make sure, by choosing the regions $\sigma$ small enough, $i$. e., by taking $\mu$ so large that the squares of the net-work will be small enough - I say, we will make sure that the boundary of each region $\sigma$ abutting on the boundary of $S$ has but a single arc of its boundary in common with the latter boundary. ${ }^{*}$ Furthermore, if $\sigma_{1}, \sigma_{2}, \ldots, \sigma_{p}$ denote the $p$ regions $\sigma$ of this latter class, then $\mu$ shall also be so large that $\sigma_{i}$ abuts only on $\sigma_{i-1}$ and $\sigma_{i+1}$, but on no other $\sigma_{k}, k=1, \cdots p$. That the removal of $\sigma_{1}$ from $S$ leaves a connected region $S_{1}$ follows from the theorem $\dagger$ that a cross-cut divides a region at most into two regions, and from the fact that the portion of the boundary of $\sigma_{1}$ not lying in the boundary of $S$ forms a cross-cut of $S$. Let $\sigma_{2}, \cdots, \sigma_{p-1}$ be next removed, thus leaving $S_{p-1}$. Proceeding now to remove from $S_{p-1}$ a region $\sigma_{p}^{\prime}$, which of course cannot be $\sigma_{p}$, we may find that $S_{p-1}$ thereby falls asunder. Nevertheless, by a suitable choice of $\sigma_{p}^{\prime}$, such an event may always be avoided. Suppose there are points $(x, y)$ in $S_{p-1}$ for which $x$, for example, is greater than it is for the points of $\sigma_{p}$. Let $X$ be the maximum value of $x$ on the boundary of $S_{p-1}$ and consider the points $(X, y)$ of this boundary. Let $Y^{p-1}$ be the maximum value of the $y$ 's here entering. Then the square of $S_{p-1}$ whose vertex is at $(X, Y)$ has at least two adjacent sides pertaining to the boundary of $S_{p-1}$, and hence its side or sides not pertaining to this boundary form a cross-cut of $S_{p-1}$. This square may, therefore, be chosen as the desired $\sigma_{p}^{\prime}$. All further details of the arithmetization are so obvious that they may be left to the reader without comment. The proof of the intermediate theorem is now complete.

Proof of Theorem (A) for the Region S. - Finally we proceed to spread out a function $f(z)$ single-valued throughout the whole region $S$ (inclusive of the boundary), developable by Taylor's

* This is, in fact, the way the regions $\sigma$ are chosen in my proof of the lemma. Each region $\sigma$ consists of a square, a part of a square, or at most a part of two squares of the net-work.

†For an arithmetic proof of this theorem cf. Mr. L.D. Ames's paper, which appears in the current volume of the BuLLETIN. 
theorem at each point of this region, and tallying with $\mathfrak{P}(z \mid a)$ in the neighborhood of the point $a$. For this purpose choose a point $a_{i}$ on the boundary of the region $\sigma_{i}(i=1,2, \ldots) ; a_{i}$ will then lie on the boundary of $S_{i-1}$ or of $S_{i}$. Connect $a_{i}$ with $a$ by a curve $\mathfrak{E}_{i}$, consisting of either part of the boundary of $S_{i-1}$ or $S_{i}$ intercepted between $a$ and $a_{i}$. Continue $\mathfrak{B}(z \mid a)$ analytically along $\mathfrak{E}_{i}$ to $\alpha_{i}$ and then define in $\sigma_{i}$ a function $f_{i}(z)$ as set forth above. Let $Z$ be a point of the common boundary of $\sigma_{i}$ and $\sigma_{i+1}$. Then I say

$$
f_{i}(Z)=f_{i+1}(Z) \text {. }
$$

For, join $a_{i}$ with $Z$ by a curve $\Gamma_{i}$ lying in $\sigma_{i}$ and meeting the boundary of $\sigma_{i}$ only in $a_{i}$ and $Z ; *$ construct similarly $\Gamma_{i+1}$. Then the four curves $\mathfrak{C}_{i}^{i}, \Gamma_{i}, \Gamma_{i+1}, \mathfrak{C}_{i+1}$ constitute a closed curve without multiple points, or else a finite number of such curves connected together either at isolated points of intersection of $\mathfrak{C}_{i}$ and $\mathfrak{E}_{i+1}$, or by lines common to both $\mathfrak{C}_{i}$ and $\mathfrak{C}_{i+1}{ }^{\dagger}$ The interior of each of these curves lies within $S$ and hence all the conditions of Theorem (A) are fulfilled for it. By virtue of the result above established $\mathfrak{P}(z \mid a)$, when continued round it, comes back to its initial value, and hence we infer without difficulty that $f_{i}(Z)=f_{i+1}(Z)$, Q.E.D.

The totality of the functions $f_{i}(z)$ thus defined constitutes, therefore, a function $f(z)$ single-valued and developable by Taylor's theorem in all points of $S$, and identically equal to $\mathfrak{P}(z \mid a)$ in the neighborhood of the point $a$. This is precisely the function $f(z)$ demanded by the theorem.

Further Theorems of Analysis which rest on Intuition.-In conclusion I desire to mention two or three further theorems of analysis which have hitherto rested on intuition. The first of these is the following: Let $S$ be a finite region of the plane. bounded by a single regular curve $C$ without multiple points. Let $O$ be a point of the plane not lying on $C$; let $P_{0}$ be a fixed point and $P$ a variable point of $C$. Let $\theta$ denote the angle $P_{0} O P$. Then, when $P$ traces out $C$, the function $\theta$, varying continuously, comes back to its initial value when $O$ is outside of $C$, and increases by $2 \pi$ or $-2 \pi$ when $O$ lies inside C.

\footnotetext{
* If, in particular, $Z$ should happen to lie on $\mathfrak{E}_{i}$, then no curve $\Gamma_{i}$ is necessary, the arc $\mathfrak{E}_{i}, \Gamma_{i}$ being replaced here merely by so much of $\mathfrak{E}_{i}$ as lies between $a$ and $a_{i}$.

†A further special case is possible, in-which no closed curves present themselves, $a$ and $Z$ being connected by a single line.

$\ddagger$ For an arithmetic definition of the angle $\theta$ and for an arithmetic proof of this theorem cf. Mr. Ames's paper above referred to.
} 
A further theorem is one whose proof may be given by the same method as that employed above for Theorem (A), namely : Let $S$ be a finite region bounded by a single regular closed curve, and let $P, Q$ be two functions, single-valued and continuous throughout the interior and boundary of $S$ and having respectively continuous partial derivations $P_{y}$ and $Q_{x}$ at all interior points. Then a necessary and sufficient condition that the value of the integral

$$
\int_{(a, b)}^{(x, y)} P d x+Q d y
$$

taken along any path lying in $S$, be independent of the path is that

$$
\frac{\partial P}{\partial y}=\frac{\partial Q}{\partial x} \text {. }
$$

In line with the arithmetic proofs here considered is the arithmetic definition of the Riemann's surface. Here the element is not the point $z$ or $(x, y)$, but the triple $(x, y, n)$, where $n$ is an integer. On the foundation of such a definition the analysis situs of the algebraic Riemann's surface * can be built up in an entirely rigorous manner-a problem which lies in the field in which Mr. Ames is now working.

Finally I will mention the following theorem : Let $T$ be any plane continuum. $\dagger$ Then there exists a set of continua $T_{1}, T_{2}, \ldots$, each of which is made up of a finite number of squares and which are so constituted that $(a)$ each contains its predecessor and (b) an arbitrary point of $T$ ultimately comes to lie within one (and hence within all later ones) of these regions. The proof of this theorem does not involve the theorem that a simple closed curve divides the plane into two regions, or the other theorems mentioned in this paper, whose proofs have hitherto rested on intuition. If it is possible, without these theorems, furthermore to show that in case $T$ is finite and is bounded by a single curve, the regions $T_{i}$ may also all be so chosen that their boundary consists of a single curve, then Theorem (A) may be proven without the use of these theorems.

We have not attempted to make a complete enumeration of

* I mean the theory laid down by Riemann in his dissertation and his papers on the Abelian functions, and set forth by Neumann in his Abel'sche Integrale, 2 ed., 1884, ch. 7.

$\dagger I$. $e$, a set of points $(x, y)$ such that $(a)$ every point of the set is an interior point and $(b)$ any two points may be connected by a regular curve lying wholly in the set. 
the theorems employed in analysis which rest on intuition and analogy. Thus no mention has been made of the theorem required to justify the ordinary use of the inner normal. But the theorems we have stated are sufficient to show in what direction further proof is necessary if the fundamental theorems of analysis are really to rest on an arithmetic basis.

HARVARD UNTVERSTTY,

Cambridge, Massachusetts, December, 1903.

\title{
ON THE THEOREM OF ANALYSIS SITUS RELAT- ING TO THE DIVISION OF THE PLANE OR OF SPACE BY A CLOSED CURVE OR SURFACE.
}

\author{
BY MR. L. D. AMES.
}

(Read before the American Mathematical Society, December 29, 1903.)

\section{The Theorem for the Plane.}

JORDAN has proved $*$ that a simple $\dagger$ closed plane curve divides the totality of the points of the plane not on the curve into an interior and an exterior region. He assumes the theorem for the polygon. For the regular curve $\dagger$ we here give a simple proof which goes back to fundamental principles, includes the polygon as a special case, and can be extended to the analogous theorems in more than two dimensions.

In two dimensions the method is as follows: Let a point be conceived as a number pair, the plane as the totality of such points, and a curve as an assemblage of points defined by one or more equations. The fundamental conception on which our proof rests is that of the order of a point. The order is a point function, uniquely defined for every point of the plane not lying on the curve, and its value is always an integer, positive, negative or zero. Before proceeding to this definition we will first define the angle $\theta$ from the line

\footnotetext{
* Jordan, Cours d'Analyse, 2d ed. 1893, vol. 1, 8, 96-102.

† A simple curve shall be defined as a continuous curve without multiple points, and a regular curve as a continuous curve such that the portion join ng any two points of the curve consists of a finite number of pieces, each of which is simple and has a continuous tangent at all its points, inclusive of its extremities. Similarly for surfaces.
} 